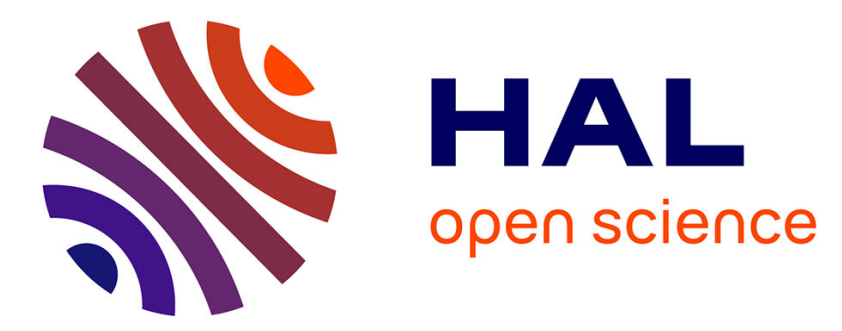

\title{
Synthèse et commande robuste d'une micropince piézoélectrique intégrée.
}

\author{
Mathieu Grossard, Nicolas Chaillet, Mehdi Boukallel, Christine
}

Rotinat-Libersa, Arnaud Hubert

\section{To cite this version:}

Mathieu Grossard, Nicolas Chaillet, Mehdi Boukallel, Christine Rotinat-Libersa, Arnaud Hubert. Synthèse et commande robuste d'une micropince piézoélectrique intégrée.. Journal Européen des Systèmes Automatisés (JESA), 2010, 44 (6), pp.661-691. 10.3166/jesa.44.661-688 . hal-00585490

\section{HAL Id: hal-00585490 \\ https://hal.science/hal-00585490}

Submitted on 13 Apr 2011

HAL is a multi-disciplinary open access archive for the deposit and dissemination of scientific research documents, whether they are published or not. The documents may come from teaching and research institutions in France or abroad, or from public or private research centers.
L'archive ouverte pluridisciplinaire HAL, est destinée au dépôt et à la diffusion de documents scientifiques de niveau recherche, publiés ou non, émanant des établissements d'enseignement et de recherche français ou étrangers, des laboratoires publics ou privés. 


\title{
Synthèse et commande robuste d'une micropince piézoélectrique intégrée
}

\author{
Mathieu Grossard* - Nicolas Chaillet ${ }^{* *}$ - Mehdi Boukallel* \\ Christine Rotinat-Libersa* — Arnaud Hubert** \\ * CEA, LIST, Interactive Robotics Laboratory, Fontenay aux Roses, F- 92265, France \\ mathieu.grossard@cea.fr \\ ** Institut Femto-ST, UMR CNRS 6174 -UFC/ENSMM/UTBM, Département Automa- \\ tique et Systèmes Micro-Mécatroniques, 24 rue Alain Savary, F-25000 Besançon
}

RÉSUMÉ. Nous avons développé une nouvelle méthode de synthèse de structures flexibles monolithiques, pour permettre la conception d'actionneurs intégrés. Notre méthode de synthèse est basée sur l'agencement optimal de blocs flexibles élémentaires, ces blocs flexibles pouvant être passifs ou rendus actifs par effet piézoélectrique inverse. Grâce à une représentation dynamique $d u$ comportement entrée( $(s) /$ sortie(s) de ces mécanismes, de nouvelles spécifications permettant de faciliter la phase d'identification et de la commande robuste des systèmes ainsi synthétisés sont prises en compte dans la méthode d'optimisation. Tirant profit de cette approche innovante, une stratégie originale de commande robuste $(H A C / L A C)$ a pu être validée en expérimentation. ABSTRACT. We have developed a new method to make the synthesis of monolithic flexible structures for designing integrated actuators. Our synthesis method is based on the optimal placement of building blocks. These elementary blocks can be made in passive materials or in active piezoelectric ceramics. Thanks to a dynamic representation of the input/output behavior of these mechanisms, some new specifications which can guaranty interesting performances for the identification and control of these systems are taken into account, from the first designing step. These last criteria helped us to draw an original design strategy for their robust control.

MOTS-CLÉS : structure flexible, piézoélectricité, optimisation topologique multi-critères, grammiens, commandabilité, observabilité.

KEYWORDS: compliant structure, piezoelectricity, multi-criteria topological optimisation, gramians, commandability, observability.

662 JESA - 44/2010. Commande dans le micromonde, pages 661 à 691 


\section{Introduction}

La mécatronique est un domaine scientifique mettant en œuvre des systèmes complexes dont la conception nécessite la connaissance de nombreuses disciplines des sciences de l'ingénieur. Elle résulte de la combinaison synergique et systémique du génie mécanique, de l'électronique, de l'automatique et de la micro-informatique, pour envisager de nouvelles façons de concevoir et de créer des systèmes plus performants. La spécificité de la conception en mécatronique est qu'elle s'appuie sur une démarche systémique à savoir, une conception simultanée et pluridisciplinaire de ces différentes entités pour rendre le système global plus performant.

Lorsque l'intégration des systèmes franchit une étape supplémentaire, la mécatronique devient adaptronique. Le terme générique adaptronique renvoie ici aux appellations plus connues sur le plan international sous les noms de smart structures, smart materials, intelligent systems, adaptive structures ou encore active structures (Hurlebausa et al., 2006). La notion d'adaptronique désigne un système (et sa démarche de développement) dans lequel tous les éléments fonctionnels d'un circuit de régulation conventionnel coexistent et dont au moins un élément est appliqué de manière multifonctionnelle (Janocha, 2007). L'existence d'éléments multifonctionnels, tels que les matériaux actifs, rend possible ce concept d'intégration fonctionnelle. Ainsi, lorsqu'on opère une réduction d'échelle aux systèmes mécatroniques rencontrés habituellement dans le macromonde, cette miniaturisation s'accompagne nécessairement d'une intégration fonctionnelle de ces systèmes. Cette tendance générale pousse les microsystèmes à posséder une densité fonctionnelle de plus en plus grande, convergeant ainsi vers le concept d'adaptronique.

Par exemple, dans le cadre applicatif de la micromanipulation, l'actionnement à base de matériaux piézoélectriques est largement privilégié pour la conception de micropince intégrée (Agnus et al., 2005). Ses nombreux avantages au sens de la conception micromécatronique, comme ses bonnes performances en terme de force, de bande passante (autorisant des dynamiques d'actionnement rapides), de compacité, en font un matériau prisé pour les dispositifs microrobotiques. Leur faible déformation mécanique (de l'ordre de $0,1 \%$ ) engendrée par les importants champs électriques (de plus de $10 \mathrm{MV} . \mathrm{m}^{-1}$ ) implique par ailleurs des résolutions nanométriques pour l'actionnement, expliquant en partie le fait que les céramiques piézoélectriques soient largement répandues dans la conception des systèmes de micromanipulation. En revanche, cette faible déformation nécessite d'imaginer des schémas d'actionnement pour maximiser les courses des actionneurs (jusqu'à quelques dizaines de micromètres) sans amoindrir de manière trop importante les forces développées à leur extrémité. La plupart des méthodes existantes dans la littérature pour synthétiser de manière systématique des structures actionnées amplificatrices visent l'emploi de structures flexibles. En effet, les mécanismes déformables monolithiques ne posent pas les problèmes d'assemblage usuels que l'on peut rencontrer lors des phases de fabrication des mécanismes et améliore la précision du mécanisme du fait de l'absence de frottement aux niveaux des articulations. Diverses méthodes topologiques de synthèse basées sur des méthodes d'optimisation pour les structures flexibles passives existent : méthode d'optimisa- 
tion de paramètres structurels (Canfield et al., 2000), de forme (Allaire et al., 2004), d'homogénéisation (Bendsœ et al., 2003), etc. Pour autant, peu de travaux traitent du problème d'optimisation multidisciplinaire de structures actives.

Nous présentons ici une nouvelle méthode de conception pseudo-optimale de microsystèmes fonctionnellement intégrés à base d'actionnement piézoélectrique. Il s'agit d'une méthode de conception préliminaire de structures flexibles à déformations réparties et actionnement distribué. Elle est basée sur l'agencement optimal de blocs flexibles élémentaires, de types réseaux de poutres, décrits par une méthode aux éléments finis dans un domaine de conception fixé. La topologie, les dimensions, les matériaux, le nombre et la position des points fixés au bâti, ceux des contacts et des actionneurs, peuvent être générés automatiquement grâce à un algorithme génétique pour fournir un ensemble de solutions pseudo-optimales vis-à-vis de critères caractérisant la tâche visée, et en correspondance avec le cahier des charges. Un logiciel, baptisé FlexIn pour Flexible Innovation, a été développé sous Matlab ${ }^{\circledR}$ pour permettre la mise en application de notre méthode. La méthodologie générale est brièvement décrite dans la deuxième section. La conception optimale, la réalisation puis la commande de telles structures intégrées nécessitent une approche du problème fortement pluridisciplinaire. Notre méthode de conception a pour vocation d'intégrer autant qu'il est possible, dès l'étape amont de conception du mécanisme, une variété de critères et pourtant tous utiles au concepteur de systèmes adaptroniques. Ces critères relèvent autant du champ de la mécanique pour satisfaire le compromis nécessaire entre rigidité et compliance que du champ de l'automatique pour permettre à terme l'identification et la commande robuste de ces systèmes flexibles. La représentation dynamique du comportement entrée-sortie des mécanismes dans la méthode permet la prise en compte de nouvelles stratégies de conception, tant du point de vue de leur topologie que de leur réponse fréquentielle en boucle ouverte. Les nouveaux critères qui en résultent sont inspirés de la théorie sur l'amortissement vibratoire des structures flexibles : ces critères visent à faciliter à terme leur identification modale, ainsi que leur commande robuste. Ces aspects font l'objet de la troisième section. La méthode de synthèse a été mise en application pour répondre au problème de l'étude de la conception d'une nouvelle micropince piézoélectrique monolithique à actionnement intégré et distribué, capable de réaliser des tâches de micromanipulation (quatrième section). Ce dispositif microrobotique est optimal vis-à-vis de critères mécaniques valables en régime statique, du type force de blocage et déplacement libre, mais répond aussi aux exigences fixées par les nouveaux critères du cahier des charges portant sur la réponse fréquentielle. Dans la dernière section, une structure est spécifiquement étudiée, puis prototypée pour valider en expérimentation la pertinence de notre approche. Pour aller jusqu'au bout de la démarche de conception en adaptronique, une commande en déflexion baptisée HAC/LAC est ensuite synthétisée, en tirant profit des propriétés de la réponse fréquentielle en boucle ouverte d'un tel système. Les performances atteintes expérimentalement avec cette approche conforte la pertinence des critères dans notre approche de conception globale par FlexIn. 


\section{FlexIn : une méthodologie stochastique pour la synthèse de mécanismes flexibles}

Cette section présente la démarche globale de la méthode pour la conception de structures flexibles. La méthode, implémentée dans un logiciel baptisé FlexIn, repose sur un algorithme génétique multicritères pour optimiser la topologie, l'actionnement et les conditions aux limites de structures flexibles décrites par un assemblage de blocs flexibles élémentaires. Ces blocs sont des réseaux de poutres, décrits par un modèle aux éléments finis. Davantage de détails concernant la méthode peuvent être trouvés dans (Bernardoni, 2004), (Grossard, 2008) et (Grossard et al., 2008).

\subsection{Blocs flexibles élémentaires}

Dans le cas d'une structure flexible constituée de poutres, il est généralement possible de la partitioner en blocs flexibles élémentaires. Pour éviter de considérer toute la combinatoire des agencements possibles de poutres, le mécanisme est sous-structuré en blocs flexibles de rigidités variables. Ces blocs sont définis comme des agencements prédéfinis de plusieurs poutres au sein d'un encombrement donné. Ils sont caractérisés par leur forme géométrique, leur matériau, leurs dimensions, puis sont assemblés de façon à constituer la structure du mécanisme déformable. Cet assemblage permet ainsi la conception finale de structures monolithiques.

Deux bibliothèques d'éléments blocs flexibles sont proposées dans FlexIn. Ces bases de blocs sont respectivement composées de 36 et 19 blocs passifs et piézoélectriquement actifs figure 11). Ces différents blocs, composés d'assemblage de poutres élémentaires, permettent la synthèse d'une grande variété de topologies.

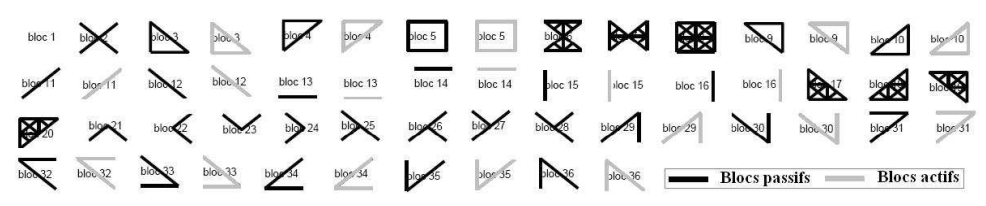

Figure 1. Bibliothèques de blocs flexibles passifs (noir) et actifs (gris) implémentées dans FlexIn

Par ailleurs, une des motivations de nos travaux est de concevoir des mécanismes monolithiques. Cela est désormais rendu possible en considérant des structures planes faites de blocs monomatériaux piézoélectriques :

- soit les blocs sont alimentés en tension pour faire office simultanément de structure et d'actionneurs intégrés ;

- soit ils ne sont pas alimentés, auquel cas ils ne jouent que le rôle de structure mécanique passive dans le comportement entrée-sortie du mécanisme. 


\subsection{Principe de la méthode}

La méthode logicielle FlexIn utilise une représentation des structures à déformations réparties qui est favorable à la fois à l'interaction avec l'opérateur, à l'optimisation, à la simulation et au prototypage. En effet, les structures flexibles y sont décrites comme un assemblage de blocs élémentaires prédéfinis. La relation mécanique entre ces éléments de base, classés en plusieurs catégories (les variables de raideurs, de conditions aux limites, de symétrie, de base, de contacts, de sorties), définit complètement le comportement entrée/sortie du mécanisme flexible. L'actionnement de la structure mécanique est réalisé par la présence soit d'actionneurs externes (en force ou en déplacement) rapportés sur la structure flexible, soit par la présence de blocs piézoélectriques actifs intégrés au sein de la structure mécanique. Le nombre et les emplacements autorisés de ces conditions aux limites sont définis par l'utilisateur de la méthode lors de la spécification du problème de conception. Des conditions aux limites en déplacement sur la structure peuvent aussi être imposées pour créer la base fixe (déplacements imposés nuls sur certains nœuds de la structure) ou prendre en compte des conditions de symétrie sur la topologie. Enfin, des comportements non linéaires dans la réponse entrée/sortie de ces structures peuvent être induits par la présence de contacts unilatéraux intermittents : leur nombre, leur jeu et leur emplacement autorisés dans la structure sont également renseignés par l'utilisateur de la méthode. Pour le moment, la méthode ne permet de traiter que la conception de structures planes dont les mouvements sont contenus dans ce même plan.

Dans la démarche de conception avec la méthode des blocs, le concepteur passe ainsi par plusieurs étapes :

- spécification d'un problème d'optimisation sur la base d'un cahier des charges ;

- traitement du problème d'optimisation par l'algorithme génétique ;

- sélection d'une solution pseudo-optimale vis-à-vis de différents critères parmi l'ensemble de solutions retournées par l'algorithme multi-objectif;

- interprétation et affinage de l'assemblage de blocs de cette structure ;

- export du modèle de la structure en vue d'autres simulations complémentaires ;

- réalisation à terme du prototypage de la solution retenue.

\subsection{Algorithme génétique multicritère}

La méthode d'optimisation stochastique utilisée pour la synthèse des structures mécaniques est celle décrite dans (Grossard, 2008) et (Grossard et al., 2008). Plusieurs fonctions-objectifs sont disponibles dans FlexIn, permettant ainsi la synthèse de mécanismes parmi un vaste cahier des charges :

- plusieurs fonctions d'évaluation d'origine mécanique peuvent être spécifiées (par exemple, maximisation du déplacement libre et/ou force de blocage à l'extrémité du mécanisme); 
- divers critères issus du champ de l'analyse modale et vibratoire ont été récemment implémentés dans FlexIn pour permettre d'atteindre a posteriori certaines contraintes spécifiques pour la commande robuste des microrobots.

À l'étape de convergence, l'algorithme d'optimisation restitue un ensemble de solutions pseudo-optimales dans le cas d'une optimisation multi-critère. Le concepteur peut alors choisir, analyser et interpréter les structures obtenues. Un logiciel éléments finis (FE) peut ensuite être utilisé pour des analyses en phase de post-traitement.

\subsection{Modèle élements finis électromécanique des structures flexibles}

Dans cette sous-section sont présentées la modélisation éléments finis (EF) 2D adoptée pour les blocs piézoélectriques de construction, ainsi que la manière dont ceux-ci sont implémentés dans la méthode d'optimisation.

\subsubsection{Poutre piézoélectrique élémentaire}

Dans FlexIn, il est fait l'hypothèse que les mécanismes flexibles sont soumis à des déformations structurelles résultant principalement de la flexion des poutres dans le plan. Ainsi, les modèles des blocs sont obtenus en considérant une formulation EF des poutres du type Navier-Bernoulli. Les paramètres structurels de chaque bloc rectangulaire sont la hauteur, la largeur et l'épaisseur. Les caractéristiques matériaux de chaque bloc sont paramètrés par le module de Young, le coefficient de Poisson, la densité, ainsi que les coefficients piezoelectriques. Pour permettre le calcul des différents critères d'optimisation, FlexIn utilise le modèle EF de chaque bloc de la bibliothèque.

Pour obtenir ce modèle, un modèle de la poutre piézoélectrique élementaire est nécessaire. Nous avons développé une formulation du problème plan appropriée à nos hypothèses. Pour plus de détails sur cette partie, le lecteur pourra se référer à (Grossard, 2008) et (Grossard et al., 2008).

\subsubsection{Blocs flexibles élémentaires}

L'assemblage de poutres piézoélectriques élémentaires permet la construction de blocs actifs de topologies variées. L'intérêt d'utiliser des blocs actifs est qu'ils permettent de coupler directement plusieurs degrés de libertés (ddl) mécaniques, autorisant ainsi des mouvements complexes au sein d'un seul élément du maillage de la structure flexible. Ce phénomène a été mis en évidence dans (Grossard et al., 2007) sur le cas de la conception d'un micro-actionneur piézoélectrique monolithique avec FlexIn.

Afin de prendre en compte l'orientation des poutres dans le plan, les matrices caractérisant le comportement électromécanique de chaque poutre sont d'abord exprimées dans la base générale, puis sont assemblées pour donner les matrices raideur $\mathbf{K}_{B l o c}$, masse $\mathbf{M}_{B l o c}$ et couplages piézoélectriques $\mathbf{G}_{B l o c}$ de chaque bloc. 


\subsubsection{Modèle éléments finis des structures piézoélectriques}

Les différentes matrices de chaque bloc sont calculées numériquement en considérant chaque combinaison des valeurs discrètes permises par les variables d'optimisation, i.e. matériaux et taille des blocs. Ainsi, le calcul de l'ensemble des matrices est fait une seule fois au début de l'algorithme, permettant ainsi de gagner en temps de calcul.

De cette structuration par blocs, il est possible de déduire le comportement dynamique global de la structure assemblage matriciel (Girard, 1997). Le modèle dynamique discrétisé du comportement non dissipatif des structures est ramené sous la forme canonique suivante :

$$
\begin{aligned}
& \mathbf{M}_{g} \ddot{\mathbf{X}}_{g}+\mathbf{K}_{g} \mathbf{X}_{g}=\mathbf{E}_{g} \mathbf{u} \\
& \mathbf{y}=\mathbf{F}_{g} \mathbf{X}_{g}
\end{aligned}
$$

Dans FlexIn, le modèle dynamique d'un mécanisme flexible est paramétré par un triplet $(p, r, s)$ d'entiers, qui définissent la taille des matrices impliquées dans l'écriture du modèle :

- $p$ pour dénombrer le nombre de degrés de liberté (au sens éléments finis) de la structure ( $p=3 \times$ Nbre nœuds);

- $r$ pour représenter le nombre de sorties mécaniques du système;

- $s$ pour caractériser le nombre d'actionneurs (données d'entrée).

Avec ces notations, il s'ensuit que :

- les vecteurs $\mathbf{X}_{g}$ et $\ddot{\mathbf{X}}_{g}$, qui représentent respectivement les déplacements et accélérations nodaux de la structure treillis, sont de dimension $p \times 1$;

- le vecteur $\mathbf{u}$, dont les composantes dépendent du temps, est de taille $s \times 1$; il définit les actions de commande sur le système. Par convention, les composantes de la matrice $\mathbf{E}_{g}$ de répartition des entrées sur le système sont calculées de sorte que la (ou les) grandeur(s) de commande soi(en)t normée(s). Dans le système d'unités propre à FlexIn, les grandeurs de commande élémentaires sont de $1 \mathrm{~V}$ dans le cas d'un actionnement par bloc actif ;

- le vecteur des sorties $\mathbf{y}$ à commander est de dimension $r \times 1$; la matrice de sortie $\mathbf{F}_{g}$ traduit la répartition des nœuds à commander. Dans une première implémentation, cette matrice, de taille $r \times p$, est uniquement constituée de 0 et de 1 . Ces derniers sont placés aux emplacements nodaux correspondants aux sorties du mécanisme et les 0 sont disposés aux emplacements des nœuds libres qui ne désignent pas de sortie mécanique.

Chaque élément de $\mathbf{u}$ (respectivement $\mathbf{y}$ ) traduisant une action de commande (respectivement d'observation) sur un actionneur (respectivement sur un capteur de sortie) de la structure est défini par une entrée non nulle dans la colonne correspondante de $\mathbf{E}_{g}$ (respectivement ligne de $\mathbf{F}_{g}$ ). 
Considérant des sorties à commander en déplacement, FlexIn est ainsi capable d'optimiser la flexibilité dynamique (cas d'un actionneur en force), ou la transmissibilité dynamique (cas d'un actionneur en déplacement) d'un mécanisme.

\section{Nouveaux critères pour l'évaluation des performances des structures en vue de leur commande}

Cette section présente les développements menés pour introduire une représentation dynamique du comportement entrée(s)-sortie(s) des mécanismes flexibles sous FlexIn, qui soit propice à la prise en compte de nouvelles stratégies de conception optimale de ces systèmes. Deux nouveaux critères numériques sont introduits dans FlexIn pour tenter de répondre, dès l'étape amont de conception du mécanisme, aux problématiques liées à l'identification et à la commande des systèmes flexibles. Ces nouveaux critères, une fois numérisés, sont ensuite mis à profit pour étudier la conception optimale d'un micro-actionneur piézoélectrique monolithique par le logiciel de synthèse FlexIn. Parmi le panel des solutions pseudo-optimales candidates au problème de conception, une structure a été choisie, puis spécifiquement étudiée en vue de son prototypage et de son test ultérieurs.

\subsection{Equations modales des structures flexibles}

Dans FlexIn, la réponse dynamique des structures est résolue par superposition modale, dans laquelle une solution de la forme :

$$
\mathbf{X}_{g}(t)=\sum_{i=1}^{p} \mathbf{Q}_{i} \mathbf{q}_{g}(t)
$$

est obtenue par une approche modes réels.

Les vecteurs $\mathbf{q}_{g}$ sont de taille $p \times 1$ et décrivent l'évolution temporelle de l'amplitude des modes vibratoires. Les vecteurs $\mathbf{Q}_{i}$ représentent les déformées modales correspondantes et forment la matrice des vecteurs modaux indépendants :

$$
\mathbf{Q}=\left[\begin{array}{lll}
\mathbf{Q}_{1} & \ldots & \mathbf{Q}_{p}
\end{array}\right]
$$

Les déformées modales et les pulsations propres associées $\omega_{i}$, qui forment une matrice diagonale diag $\left(\omega_{i}^{2}\right)$, sont obtenues par résolution du problème libre aux valeurs propres :

$$
\left(\mathbf{K}_{g}-\omega_{i}^{2} \mathbf{M}_{g}\right) \mathbf{Q}_{i}=0
$$

Dans la suite, les $p$ pulsations propres sont rangées conventionellement par ordre croissant $\omega_{1}^{2} \leq \omega_{2}^{2} \leq \ldots \omega_{p}^{2}$. 
Le changement de variables des coordonnées physiques aux coordonnées modales

$$
\mathbf{X}_{g}=\mathbf{Q q}_{g}
$$

peut être opéré dans l'équation [1]. En pré-multipliant par $\mathbf{Q}^{t}$ cette équation dynamique, les relations d'orthogonalité conduisent au nouveau système écrit dans la base modale :

$$
\begin{aligned}
& \ddot{\mathbf{q}}_{g}+\operatorname{diag}\left(\omega_{i}^{2}\right) \mathbf{q}_{g}=\mathbf{Q}^{t} \mathbf{E}_{g} \mathbf{u} \\
& \mathbf{y}=\mathbf{F}_{g} \mathbf{Q} \mathbf{q}_{g}
\end{aligned}
$$

En choisissant une matrice d'amortissement diagonale dans la base des modes propres, les structures FlexIn possèdent un modèle dynamique amorti qui se présente sous la forme du doublet d'équations matricielles suivant :

$$
\begin{aligned}
& \ddot{\mathbf{q}}_{g}+\operatorname{diag}\left(2 \xi_{i} \omega_{i}\right) \dot{\mathbf{q}}_{g}+\operatorname{diag}\left(\omega_{i}^{2}\right) \mathbf{q}_{g}=\mathbf{b}_{g}^{t} \mathbf{u} \\
& \mathbf{y}=\mathbf{c}_{g} \mathbf{q}_{g}
\end{aligned}
$$

dans lequel $\mathbf{b}_{g}=\left[\mathbf{b}_{1} \ldots \mathbf{b}_{p}\right]$ de dimension $s \times p$ et $\mathbf{c}_{g}=\left[\mathbf{c}_{1} \ldots \mathbf{c}_{p}\right]$ de dimension $r \times p$ sont définis à partir des vecteurs réels :

$$
\begin{aligned}
\mathbf{b}_{i} & =\mathbf{E}_{g}^{t} \mathbf{Q}_{i} \\
\mathbf{c}_{i} & =\mathbf{F}_{g} \mathbf{Q}_{i}
\end{aligned}
$$

$\mathbf{b}_{i} \in \mathbb{R}^{s}$ (respectivement $\mathbf{c}_{i} \in \mathbb{R}^{r}$ ) définit la $i^{e}$ colonne de $\mathbf{E}_{g}^{t} \mathbf{Q}$ (respectivement $\mathbf{F}_{g} \mathbf{Q}$ ).

\subsection{Représentation d'état associée}

En accord avec (Lim et al., 1993), un vecteur d'état modal $\mathbf{x}$ de dimension $N \times 1$ (où $N=2 p$ ) peut être choisi tel que :

$$
\mathbf{x}=\left(\begin{array}{lllll}
\dot{\mathbf{q}}_{g_{1}} & \omega_{1} \mathbf{q}_{g_{1}} & \ldots & \dot{\mathbf{q}}_{g_{p}} & \omega_{p} \mathbf{q}_{g_{p}}
\end{array}\right)^{t}
$$

avec l'avantage que les composantes du vecteur d'état possèdent toutes la même unité, contrairement à un choix plus classique du type position et vitesse (Hac et al., 1993). Dans ce choix de vecteur d'état, $\omega_{i}$ représente la pulsation du $i^{e}$ mode et $\mathbf{q}_{g_{i}}$ le vecteur de déplacement modal associé. Le triplet de matrices $(\mathbf{A}, \mathbf{B}, \mathbf{C})$ de la représentation d'état

$$
\begin{aligned}
& \dot{\mathbf{x}}=\mathbf{A x}+\mathbf{B u} \\
& \mathbf{y}=\mathbf{C x}
\end{aligned}
$$


qui est associée à ce choix de vecteur s'écrit :

$$
\begin{aligned}
& \mathbf{A}=\operatorname{diag}\left(\mathbf{A}_{1}, \ldots, \mathbf{A}_{p}\right) \\
& \mathbf{B}=\left(\mathbf{B}_{1}^{t}, \ldots, \mathbf{B}_{p}^{t}\right)^{t} \\
& \mathbf{C}=\left(\mathbf{C}_{1}, \ldots, \mathbf{C}_{p}\right)
\end{aligned}
$$

avec, pour $i=1, \ldots, p$,

$$
\mathbf{A}_{i}=\left[\begin{array}{cc}
-2 \xi_{i} \omega_{i} & -\omega_{i} \\
\omega_{i} & 0
\end{array}\right], \quad \mathbf{B}_{i}=\left[\begin{array}{c}
\mathbf{b}_{i}^{t} \\
\mathbf{0}_{1 \times s}
\end{array}\right], \quad \text { et } \quad \mathbf{C}_{i}=\left[\begin{array}{ll}
\mathbf{0}_{r \times 1} & \frac{1}{\omega_{i}} \mathbf{c}_{i}
\end{array}\right]
$$

La matrice d'état $\mathbf{A}$ est implicitement fonction des caractéristiques de certains paramètres de spécifications de la structure mécanique (comme la configuration, la topologie, le couple matériau(x)/épaisseur). La matrice de commande $\mathbf{B}$ est plus directement liée au type et à l'emplacement des actionneurs, et la matrice d'observation $\mathbf{C}$ à l'emplacement de la sortie mécanique observée.

\subsection{Un nouveau critère pour l'évaluation de la qualité de la réduction de modèle : $\mathcal{J}_{1}^{k}$}

\subsubsection{Problématique de l'identification modale}

Dans les coordonnées physiques, le nombre de degrés de liberté d'un modèle discrétisé de structure par la méthode EF est généralement très important et ce, d'autant plus que la géométrie laisse apparaître un nombre de nœuds important. Cependant, pour la commande, et notamment pour l'utilisation des méthodologies de synthèse modernes, il reste indispensable de se ramener à des modèles identifiés d'ordre le plus faible possible. La réduction de modèle semble donc être la seule solution pour se ramener à un système d'ordre raisonnable.

La technique de la troncature dans la base de représentation modale peut être un moyen de réduire de manière drastique le nombre de degrés de liberté contribuant effectivement à la réponse du mécanisme. Cette technique impose de calculer la matrice de transfert $\mathbf{G}$ en fonction de la variable de Laplace s,

$$
\mathbf{y}(s)=\mathbf{G}(s) \mathbf{u}(s)
$$

qui lie les entrée(s)-sortie(s) du système par une décomposition en matrices de transfert modales.

La réponse continue $\mathbf{y}$ de la structure à une excitation harmonique stationnaire $\mathbf{u}$ est également harmonique de même fréquence, puisque le système est considéré linéaire. 
La matrice des fonctions de transfert de la structure dans les coordonnées modales est :

$$
\mathbf{G}(s)=\mathbf{C}(s \mathbf{I}-\mathbf{A})^{-1} \mathbf{B}
$$

Sa décomposition en fonctions de transfert modales devient :

$$
\mathbf{G}(s)=\sum_{i=1}^{p} \mathbf{G}_{i}(s)
$$

où

$$
\mathbf{G}_{i}(s)=\mathbf{C}_{i}\left(s \mathbf{I}-\mathbf{A}_{i}\right)^{-1} \mathbf{B}_{i}=\frac{\mathbf{c}_{i} \mathbf{b}_{i}^{t}}{s^{2}+2 \xi_{i} \omega_{i} s+\omega_{i}^{2}}
$$

est la matrice de fonctions de transfert du $i^{i}$ mode (Gawronski, 2003).

A partir de l'expansion modale du transfert entrée(s)-sortie(s), la troncature modale peut s'avérer être une opération délicate. Elle passe nécessairement par un choix à faire sur le compromis entre l'ordre du modèle tronqué et sa précision de représentation du modèle complet. Sans que l'ordre ne deviennent trop élevé, il faut veiller à ce que le contenu fréquentiel ne soit pas insuffisant en ne retenant qu'un nombre trop faible de modes.

Pour éviter ce risque, il est nécessaire de conduire les simulations avec un modèle de structure (modèle de simulation) le plus fin possible, qui inclut tous les modes possédant une contribution significative dans la réponse du système. Ainsi, un nouveau critère $\mathcal{J}_{1}^{k}$ est implémenté dans FlexIn.

\subsubsection{Réduction de Moore dans la base modale}

La base dite équilibrée est définie comme celle dans laquelle les grammiens de commandabilité $\mathbf{W}_{C}$ et observabilité $\mathbf{W}_{O}$ associés à la représentation d'état sont diagonaux et égaux

$$
\mathbf{W}_{C}=\mathbf{W}_{O}=\mathbf{W}=\operatorname{diag}\left(\begin{array}{ccc}
\sigma_{1} & \ldots & \sigma_{N}
\end{array}\right)
$$

où les scalaires $\sigma_{i}$ sont appelés valeurs singulières de Hankel, notées HSV dans la suite. Ces termes reflètent les commandabilité et observabilité jointes des variables d'état dans la base équilibrée.

Par ailleurs, la technique de Moore (Moore, 1981), qui consiste à éliminer les états peu commandables et observables, se révèle très précieuse lorsqu'il est nécessaire d'élaborer un modèle réduit. Les $H S V$ sont donc une bonne indication de la précision du modèle réduit.

Dans le cas spécifique des structures flexibles, les $H S V$ peuvent être rapidement approchées par l'expression suivante :

$$
\sigma_{i}=\frac{\left\|b_{i}\right\|_{2}\left\|c_{i}\right\|_{2}}{4 \xi_{i} \omega_{i}^{2}}
$$




\subsubsection{Ecriture du critère numérique $\mathcal{J}_{1}^{k}$}

Dès l'étape de conception du système flexible, la réduction optimale du modèle dynamique peut être prise en compte dans FlexIn grâce à un nouveau critère $\mathcal{J}_{1}^{k}$. Par son implémentation logicielle, ce critère numérique doit répondre à une double exigence :

- d'une part, il doit permettre de laisser le concepteur libre de choisir le nombre $k$ de modes vibratoires dominants de la structure;

- d'autre part, il doit permettre de quantifier l'erreur causée par l'approximation du modèle complet en un modèle tronqué aux $k$ premiers modes.

Les autorités de contrôle sur les $k$ premiers modes doivent ainsi être maximisées. À l'inverse, celles sur les modes plus haute fréquence situés hors de la bande d'inté-

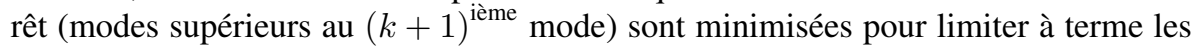
risques de déstabilisation (spillover). Ces états modaux caractérisés par de faibles $H S V$ sont faiblement commandables et observables et peuvent ainsi être écartés du modèle. Le critère $\mathcal{J}_{1}^{k}$ proposé, qui doit être maximisé, prend ainsi la forme suivante :

$$
\mathcal{J}_{1}^{k}=\frac{\sum_{i=1}^{k} \sigma_{i}}{\sum_{i=k+1}^{p} \sigma_{i}}
$$

où les $\sigma_{i}$ sont ordonnées selon les modes $\omega_{i}$ croissants.

La définition de la norme infinie $\|.\|_{\infty}$ peut permettre une interprétation graphique du critère : $\sigma_{i}$ est directement proportionnelle à l'amplitude du pic de résonance

$$
\left\|G_{i}\right\|_{\infty} \simeq 2 \sigma_{i}
$$

ce qui laisse augurer par l'utilisation du critère $\mathcal{J}_{1}^{k}$ une forme de la réponse fréquentielle de la structure telle que décrite à la figure 2.

\subsection{Un nouveau critère pour forcer la propriété d'alternance pôles/zéros : $\mathcal{J}_{2}^{k^{\prime}}$}

\subsubsection{Problématiques liées à la commande}

Les imperfections des techniques de représentation des structures flexibles sont nombreuses et se retrouvent lors de leur commande.

La trop grande schématisation du comportement structural des matériaux, les non linéarités mécaniques et les variations locales de géométrie sont autant de sources possibles qui peuvent affecter les modes flexibles d'une structure. De même, les imperfections des réalisations des encastrements peuvent modifier la nature des conditions aux limites et par là-même le comportement global du système. Ainsi, les performances atteignables sont limitées (Vincent, 1996) : 


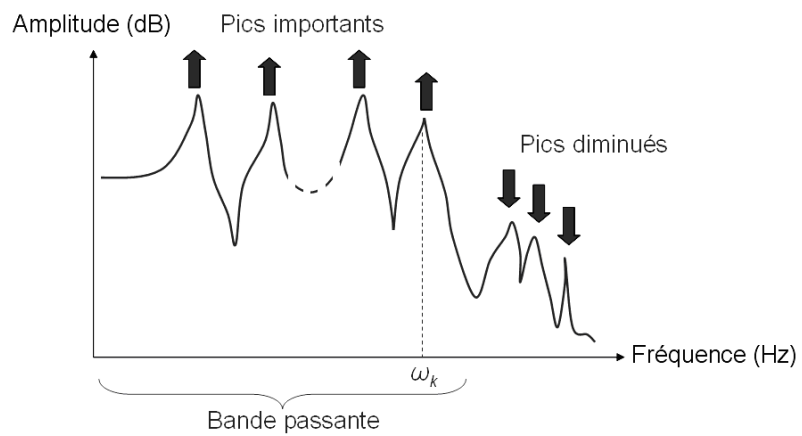

Figure 2. Forme de la réponse fréquentielle en amplitude d'un système dans le cas SISO favorisée par l'utilisation du critère $\mathcal{J}_{1}^{k}$ : l'amplitude des pics de résonance est maximisée dans la fenêtre fréquentielle $\left[0 ; \omega_{k}\right]$ et est minimisée sur $\left[\omega_{k} ;+\infty\right]$
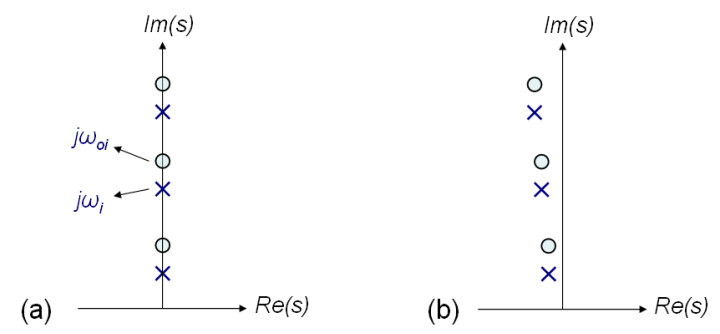

Figure 3. Lieux des pôles $(\times)$ et des zéros $(\circ)$ dans le plan complexe d'un système à alternance pôles/zéros : (a) cas non amorti; (b) cas légèrement amorti; (seule la partie supérieure du plan complexe est représentée, la figure étant symétrique par rapport à l'axe réel)

- par la qualité du modèle ou la capacité de la commande à prendre en compte convenablement les incertitudes de modélisation ;

- par la complexité de la loi synthétisée et la puissance de calcul des processeurs permettant sa mise en œuvre sur le système physique.

Pour certaines classes de systèmes flexibles, qui peuvent être modélisés comme des systèmes résonants présentant une alternance de pôles et de zéros, il existe des propriétés de robustesse naturelle de la commande vis-à-vis des incertitudes paramétriques et des dynamiques non modélisées. Cette approche conduit aussi à des lois de commande simples à calculer et à implémenter qui consistent à amortir les modes dominants (Aphale et al., 2007). Ces techniques de commande amènent également un gain en robustesse qu'il est possible d'exploiter pour stabiliser a posteriori une boucle de compensation globale assurant les performances de suivi de consigne (Missiles et al., 1983). 
L'analyse des propriétés des structures flexibles sur le plan fréquentiel a permis de proposer un nouveau critère d'optimisation pour notre méthode de conception. Ce critère noté $\mathcal{J}_{2}^{k^{\prime}}$ vise à forcer artificiellement le comportement d'alternance pôles/zéros.

\subsubsection{Ecriture du nouveau critère numérique $\mathcal{J}_{2}^{k^{\prime}}$}

L'avantage de l'approche précédente est de garantir une certaine stabilité et une robustesse vis-à-vis des changements de propriétés de la structure tant que l'alternance des pôles et des zéros est préservée. Dès lors, il semble intéressant du point de vue de la commande de ces systèmes d'implémenter un critère capable de forcer cette propriété d'alternance pôle/zéro, au moins dans la bande fréquentielle d'intérêt choisie par le concepteur pour réaliser à terme la commande. L'analyse menée précédemment a montré que cette propriété était directement liée au signe des résidus modaux.

En s'inspirant de (Martin, 1978), le nouveau critère implémenté dans FlexIn pour forcer la contrainte d'alternance pôle/zéro, et qui doit être maximisé, s'écrit :

$$
\mathcal{J}_{2}^{k^{\prime}}=\left|\sum_{i=1}^{k^{\prime}} \operatorname{sign}\left(\mathbf{c}_{i}(a) \mathbf{b}_{i}^{t}(l)\right)\right|
$$

où $\operatorname{sign}(\cdot)=\{-1 ; 0 ;+1\}$ selon le signe de l'argument. La somme sur $i$ s'étend à tous les modes appartenant à la bande fréquentielle dans laquelle l'alternance est souhaitée. $\mathbf{c}_{i}(a) \mathbf{b}_{i}^{t}(l)$ désigne le résidu du $i^{e}$ mode pour la sortie $a$ et l'entrée $l$.

\section{Synthèse multi-objectif d'une micropince piézoélectrique}

La méthode de synthèse a été mise à profit pour concevoir une structure microrobotique monolithique piézoélectrique capable de réaliser la fonction de préhension. Il s'agit d'une micropince faite de deux doigts de serrage symétriques et à mobilité indépendante. Les deux nouveaux critères $\mathcal{J}_{1}^{k}$ et $\mathcal{J}_{2}^{k^{\prime}}$, lorsqu'ils sont utilisés simultanément, fournissent des informations intéressantes sur les performances du système qu'il sera possible d'atteindre en boucle fermée, grâce à leur comportement fréquentiel en boucle ouverte.

\subsection{Spécifications du problème d'optimisation}

La topologie du doigt-actionneur gauche est choisie comme paramètre d'optimisation. Elle est définie par l'assemblage de blocs élémentaires actifs et/ou passifs dans la $(2 \times 2)$-matrice de maillage, sous les contraintes dimensionnelles des blocs données par sur la figure 4. L'évaluation des critères par FlexIn sera effectuée lorsque le système est soumis à une différence de potentiel de $200 \mathrm{~V}$ entre les électrodes supérieure et inférieure des blocs actifs. À l'inverse, les propriétés électromécaniques des blocs passifs (qui ne sont pas alimentés en tension électrique) ne seront pas exploitées. Seules leurs caractéristiques mécaniques pures (rigidité, masse) interviendront dans le 
comportement statique et fréquentiel de la microstructure. Le nombre de blocs actifs de la structure est laissé libre au cours de l'optimisation, compris entre 1 et 4 .

La différence de potentiel $V_{\text {gauche }}$ doit induire une déformation globale du doigt de préhension fournissant :

- un mouvement d'ouverture et de fermeture ;

- l'application d'une force de réaction $F_{m}$ sur l'objet saisi dans la direction de l'axe $\mathbf{x}_{R}$ (figure 4 ).

Enfin, les nœuds à la base de la structure-treillis définissent les conditions aux limites d'encastrement et peuvent être laissés, tous ou en partie, bloqués en déplacements et rotation par l'optimisation (figure 4).

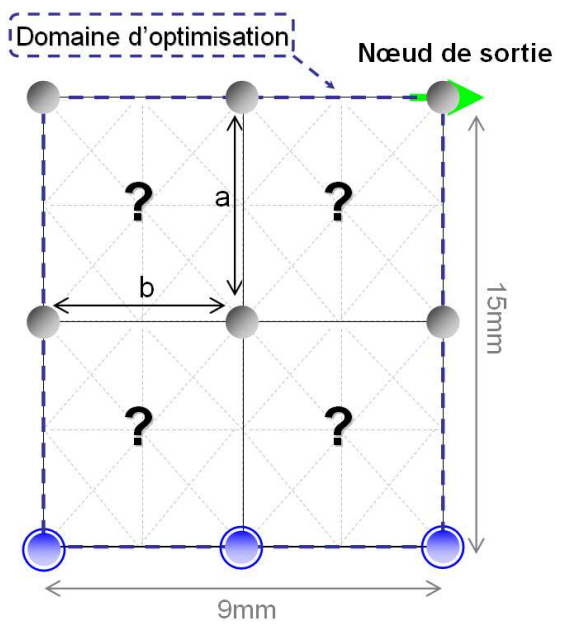

Légende:
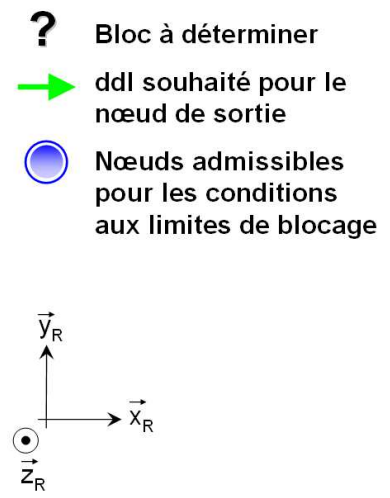

Figure 4. Spécifications des paramètres du problème d'optimisation pour le doigt de préhension gauche

Deux premiers critères mécaniques relatifs aux performances du système en régime quasi statique sont considérés au cours de l'optimisation multi-critère :

- la maximisation de la déflexion $\delta$ selon la direction $\mathbf{x}_{R}$ permet de tendre vers une amplification des faibles déplacements inhérents à la nature de l'actionnement piézoélectrique ;

- la force de réaction mécanique à sortie bloquée est maximisée au nœud de sortie pour permettre d'atteindre des performances adaptées à une tâche de micromanipulation.

Par ailleurs, les critères $\mathcal{J}_{1}^{k}$ et $\mathcal{J}_{2}^{k^{\prime}}$, nouvellement implémentés dans FlexIn, sont considérés simultanément aux deux critères mécaniques statiques précédents. Dans la présente étude, le nombre $k$ de modes dominants est choisi égal à 2 et l'alternance est également souhaitée jusqu'à ce même second mode, $k^{\prime}=2$. Après simulations, ces choix sur $k$ et $k^{\prime}$ apparaissent comme un compromis intéressant entre le cas restrictif 


\begin{tabular}{|c||c|}
\hline Critères évalués & Solution \\
\hline Déplacement libre $\delta(\mu \mathrm{m})$ & 10,69 \\
\hline Force de blocage $(N)$ & 0,84 \\
\hline $\mathcal{J}_{1}^{2}$ & 5842,35 \\
\hline $\mathcal{J}_{2}^{2}$ & 2 \\
\hline
\end{tabular}

Tableau 1. Performances théoriques évaluées par FlexIn (pour une différence potentiel électrique $V_{\text {gauche }}=200 \mathrm{~V}$ )

$k=1$ qui limite la combinatoire du problème et le cas où le nombre excessif de modes dominants impliquerait a posteriori l'obtention d'un modèle d'état d'ordre élevé.

Dans ces conditions, le critère discret $\mathcal{J}_{2}^{2}$ ne peut prendre que deux valeurs numériques. La première valeur est $\mathcal{J}_{2}^{2}=2$ et correspond à la maximisation du critère. La seconde est $\mathcal{J}_{2}^{2}=0$ et caractérise la non-alternance des deux premières résonances avec une antirésonance. Cette dernière valeur est à éviter.

Les valeurs d'amortissements modaux du système seront arbitrairement choisis égaux à $1 \%$. L'hypothèse de structures faiblement amorties est ainsi respectée, laissant l'écriture des critères $\mathcal{J}_{1}^{2}$ et $\mathcal{J}_{2}^{2}$ valide.

L'algorithme d'optimisation stoppe lorsqu'aucune nouvelle solution au problème n'est trouvée pendant plus de 135 générations.

\subsection{Exemple d'une structure pseudo-optimale de micropince piézoélectrique}

Parmi le panel de solutions pseudo-optimales obtenues, une solution en particulier est étudiée dans la suite. La structure considérée est caractérisée par de très bonnes propriétés mécaniques, ainsi que de bonnes valeurs pour les critères $\mathcal{J}_{1}^{2}$ et $\mathcal{J}_{2}^{2}$ (Table 1). La topologie correspondante de la structure est celle représentée sur la figure 5 et un prototype est présenté sur la figure 6

\section{Identification du comportement tension/déflexion}

Cette section porte sur l'étude et l'identification du micro-actionneur piézoélectrique conçu précédemment par la méthode de synthèse. L'analyse de la non-linéarité de type hystérésis propre au phénomène de la piézoélectricité a permis d'établir un modèle dynamique complet du microsystème en tension-déflexion pour permettre à terme sa commande. 


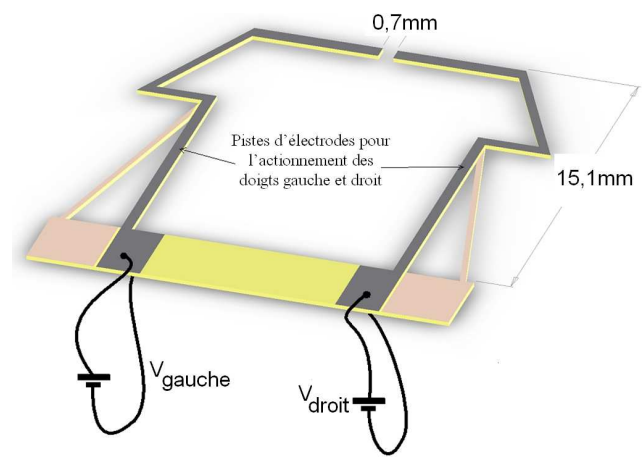

Figure 5. Vue 3D de la micropince piézoélectrique montrant les pistes d'électrodes supérieures pour les doigts gauche et droit

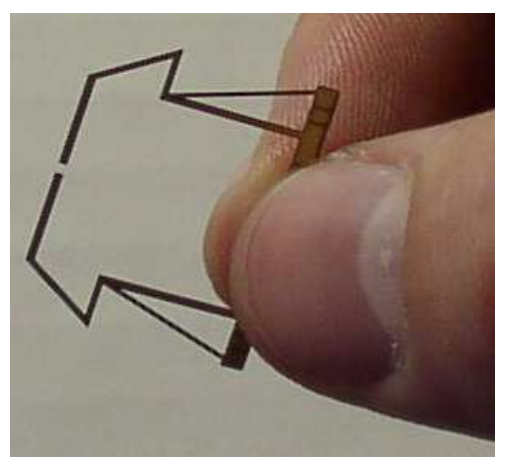

Figure 6. Photographie du prototype de la micropince usinée par découpe laser

\subsection{Banc expérimental}

Dans cette section, le modèle électromécanique reliant la tension $(V)$ et la déflexion $\left(\delta_{x}=\delta\right)$ du prototype piézoélectrique est analysé puis identifié à partir de mesures expérimentales. En particulier, les phénomènes non linéaires d'hystérésis et de dérive sont introduits dans l'écriture du modèle complet en vue de l'étape avale de commande.

Le micro-actionneur qui constitue la demi-pince est encastré à sa base dans des mors conducteurs en cuivre, et placé sur une table de micropositionnement $x-y-z$. La structure piézoélectrique nécessitant de fortes tensions pour permettre son actionnement, le système est connecté à la sortie d'un amplificateur de tension (référence $L P A$ $400, N 4 L$ ) de gain $\times 50$ et de large bande passante. Le déplacement résultant selon la direction $x$ est mesuré via un capteur laser de résolution 0, $01 \mu \mathrm{m}$ (référence Keyence $L K-G 10$ ), dont la sortie analogique est transmise vers un filtre antirepliement de fonction de transfert notée $F(s)$.

L'acquisition de la sortie en tension du filtre est réalisée grâce à un ordinateur équipé de Matlab/Simulink, ainsi que d'une carte d'acquisition NI Labview PXI, dont la fréquence d'échantillonnage vaut $\omega_{e}=2 \times \pi \times 20 \mathrm{kHz} \simeq 125 \mathrm{krad} / \mathrm{s}$ (figure 7).

\subsection{Modélisation de l'hystérésis dynamique}

La modélisation recherchée du comportement en tension/déflexion de la microstructure active doit, selon (Rakotondrabe, 2006) :

- prendre en compte les phénomènes d'hystérésis présents dans le prototype piézoélectrique ; 


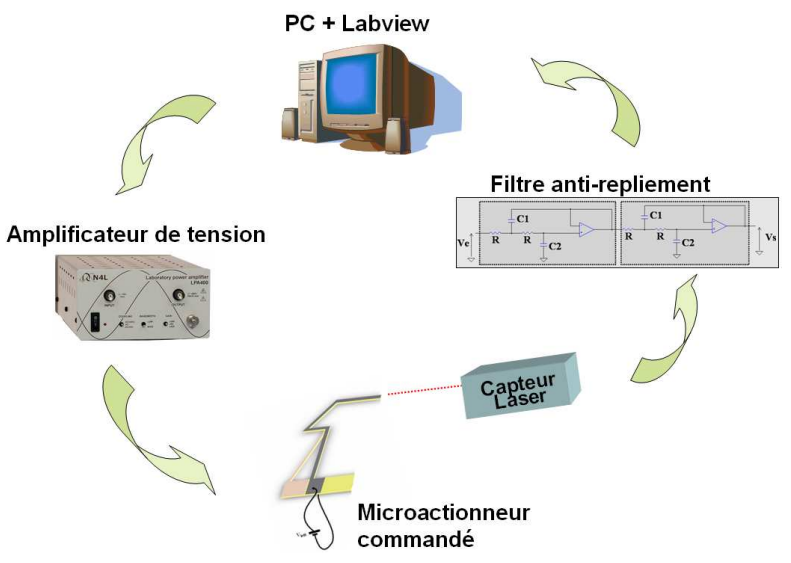

Figure 7. Schéma de la chaîne d'instrumentation

- rester simple pour les phases d'identification et de commande;

- mener à une consommation faible en coût de calculs en vue de l'implémentation logicielle.

Conformément à (Pons, 2005), nous adoptons une modélisation très générale du comportement dynamique de la déflexion. Si on omet l'effet de la force mécanique externe, cette modélisation lie de manière non linéaire la déflexion $\delta$ aux grandeurs externes que sont la tension électrique d'actionnement $U$ selon :

$$
\delta(t)=\Gamma(U, t)
$$

L'opérateur $\Gamma$ exprime la réponse de la déflexion $\delta$ en fonction de la contribution de l'hystérésis dynamique (en absence de dérive) (figure 8).

L'hystérésis dynamique, dont l'opérateur non linéaire est noté $H_{d}$ dans la suite, est souvent considéré comme la cascade en série d'un opérateur d'hystérésis statique, noté $H_{s}$ et d'un second opérateur caractérisant la partie dynamique, noté $D$ (Croft et al., 1998) (Dimmler et al., 1999) (figure 9). L'opérateur $D(t)$ est supposé linéaire, et on note dans la suite $D(s)$ sa fonction de transfert, obtenue par transformée de Laplace de la réponse impulsionnelle de $D(t)$ et caractérisée par $D(s=0)=1$.

\subsection{Identification de la dynamique}

Le comportement dynamique de la structure est identifié grâce à une étude harmonique. Pour isoler le comportement vibratoire du micro-actionneur du phénomène de dérive, l'analyse fréquentielle débute à $2 \times \pi \times 10 \mathrm{~Hz} \simeq 63 \mathrm{rad} / \mathrm{s}$. Par ailleurs, l'amplitude de la tension d'excitation est maintenue volontairement basse $(5 \mathrm{~V})$ pour 

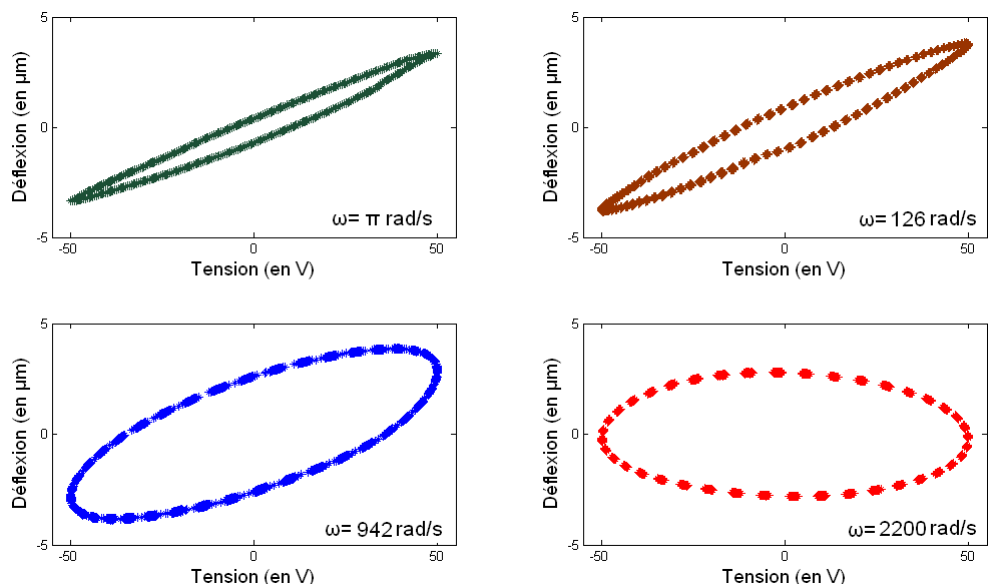

Figure 8. Observation de l'hystérésis du doigt d'actionnement gauche en régime harmonique pour différentes valeurs de fréquence

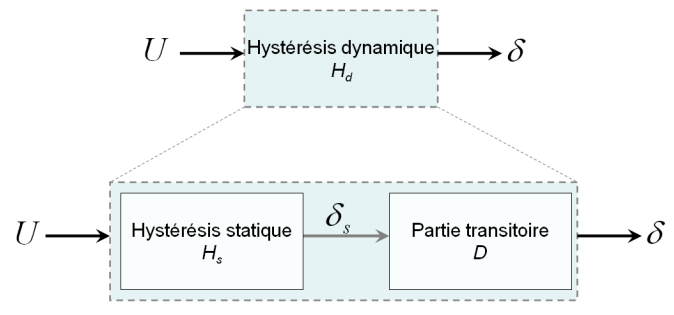

Figure 9. Equivalence comportementale de l'hystérésis dynamique

rester dans les approximations du comportement linéaire de la structure hystérétique. La déflexion $\delta$ est mesurée par le capteur de déplacement laser pendant qu'une tension sinusoïdale de fréquence variable est appliquée aux bornes du système. Un analyseur de spectre (référence HP3562A) recueille les données au tracé du diagramme de Bode.

Comme attendu par la méthode de conception, d'une part les deux premiers modes résonants sont bien dominants sur les modes résiduels hautes-fréquences, et d'autre part l'alternance désirée des pôles et zéros est maintenue dans le spectre d'intérêt. L'identification fréquentielle est donc réalisée en considérant ces deux modes flexibles seulement, impliquant de ce fait l'obtention d'un modèle réduit d'ordre 4 :

$$
D(s)=\frac{N(s)}{M(s)}
$$


où

$$
\begin{aligned}
N(s)= & 3,522 \times 10^{-7} \mathrm{~s}^{2}+2,291 \times 10^{-5} \mathrm{~s}+1 \\
M(s)= & 1,113 \times 10^{-14} \mathrm{~s}^{4}+3,499 \times 10^{-12} \mathrm{~s}^{3}+4,206 \times 10^{-7} \mathrm{~s}^{2} \\
& +3,178 \times 10^{-5} \mathrm{~s}+1
\end{aligned}
$$

Au dénominateur $M(s)$, les pulsations $\omega_{n i}$ et les amortissements $\xi_{i}$ ont été déterminées à partir des relevés expérimentaux Tableau 5.3 .

\begin{tabular}{cc|cc}
\hline \hline$\xi_{1}$ & $\omega_{n 1}\left(\right.$ rad.s $\left.^{-1}\right)$ & $\xi_{2}$ & $\omega_{n 2}\left(\right.$ rad.s $\left.^{-1}\right)$ \\
\hline $1,97 \%$ & 1597,1 & $2,12 \%$ & 5934,1 \\
\hline \hline
\end{tabular}

Tableau 2. Paramètres modaux identifiés de la fonction de transfert $D(s)$ identifiée (Pour indication, $\omega_{n 1}$ correspond à une fréquence de 254, $1 \mathrm{~Hz}, \omega_{n 2}$ à 944, $4 \mathrm{~Hz}$ )

La comparaison de la réponse fréquentielle $D(s) \cdot F(s)$ identifiée avec celle observée par l'expérimentation indique que le modèle du quatrième ordre traduit convenablement les premières dynamiques vibratoires, avant la phase de roll-off débutant à $2 \times \pi \times 1,5 \mathrm{kHz} \simeq 9,4 \mathrm{krad} / \mathrm{s}$ (figure 10 ).
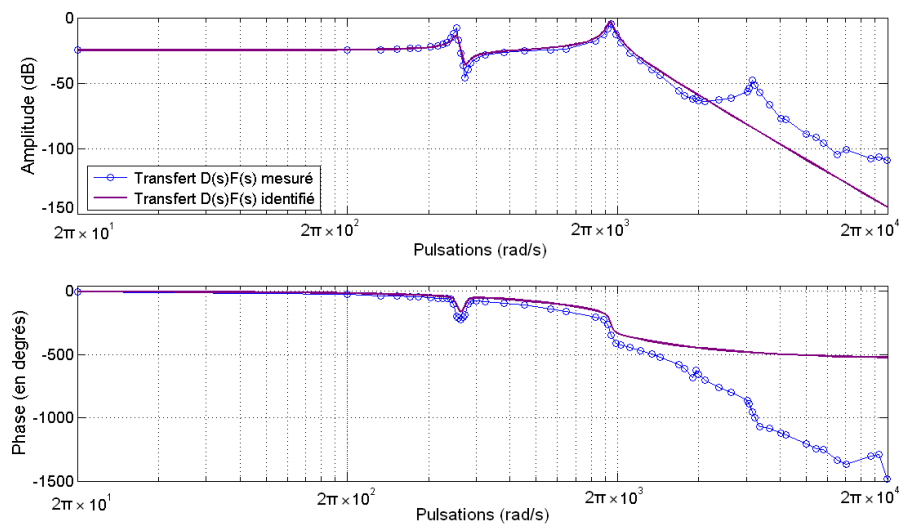

Figure 10. Diagramme de Bode expérimental du transfert en déflexion $\frac{\delta}{U}\left(\delta=\delta_{x}\right.$ en $\mu m$ et $U$ en Volt) et transfert identifié correspondant $D(s) F(s)$

\subsection{Identification de l'hystérésis statique}

La modélisation adoptée pour la partie représentant l'hystérésis statique est l'approche quadrilatérale décrite dans (Rakotondrabe, 2006). L'hystérésis $H_{s}$ est ap- 
proximé par un modèle pseudo-linéaire de pente nominale $\alpha_{0}$ et d'ordonnée à l'origine $\delta_{H}$ selon :

$$
\delta=\alpha_{0} U+\delta_{H}
$$

dans lequel $\alpha_{0}$ est calculé comme la moyenne arithmétique des pentes minimale $\alpha_{m}$ et maximale $\alpha_{M}$ des quatre droites constituant le quadrilatère (figure 11). Par ailleurs, la pente moyenne $\alpha_{0}$ et le rayon $\alpha_{E}$ sont également introduits comme suit :

$$
\left\{\begin{array}{l}
\alpha_{0}=\frac{\alpha_{M}+\alpha_{m}}{2} \\
\alpha_{E}=\frac{\alpha_{M}-\alpha_{m}}{2}
\end{array}\right.
$$

L'information sur la valeur du rayon $\alpha_{E}$ permet de quantifier l'erreur d'incertitude sur le modèle réel du système à un instant donné. Finalement, le système réel est caractérisé en statique comme un système linéaire soumis à une perturbation additive en entrée :

$$
\left\{\begin{array}{l}
\delta_{s}=\alpha_{\text {system }} U+\delta_{H} \\
\alpha_{0}-\alpha_{E} \leq \alpha_{\text {system }} \leq \alpha_{0}+\alpha_{E}
\end{array}\right.
$$

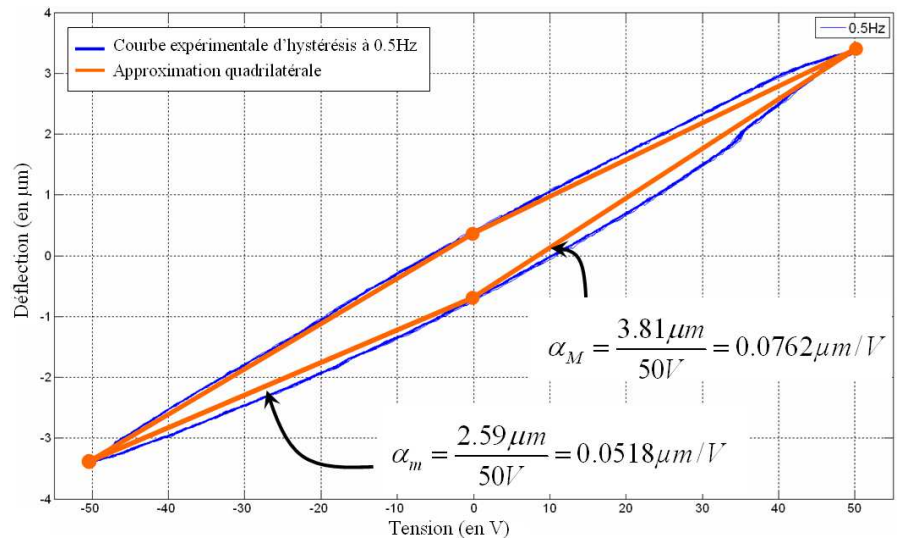

Figure 11. Identification des paramètres de la courbe d'hystérésis quadrilatérale (les segments de droites sont choisis aux nœuds du ventre de l'hystérésis, où l'amplitude de la déflexion est maximale)

\section{Synthèse d'une loi de commande HAC/LAC}

L'approche suivante, fondée à l'origine par la société Lockheed, est basée sur une commande hiérarchisée High Autorithy Control/Low Autorithy Control (HAC/LAC) (Aubrun et al., 1984); (Preumont, 2002). La stratégie de commande utilisée dans l'étude est la combinaison de deux boucles de rétroactions (figure 12). 
- la boucle interne d'amortissement réduit l'amplitude de la réponse fréquentielle au voisinage des résonances et, par conséquent, le temps de réponse à des commandes indicielles ou impulsionnelles en sortie du compensateur $H_{L}(s)$;

- le compensateur HAC, de transfert $H_{L}(s)$, synthétisé sur la base du système précédemment amorti par $H_{l}(s)$, réalise notamment l'action intégrale nécessaire en basse fréquence et traite le compromis performance-robustesse-stabilité.

\subsection{Synthèse du régulateur LAC}

La technique du PPF (Positive Position feedback) utilise un filtre du second ordre, réglé sur un mode particulier à amortir. Des performances en boucle fermée stable peuvent être atteintes en rebouclant positivement le signal mesurant la position de la déflexion sur l'entrée du compensateur. Dans l'étude, l'intérêt est centré sur l'amortissement du premier mode vibratoire $\omega_{n 1}$, l'amplitude du second mode étant de fait atténuée de $-40 \mathrm{~dB} /$ décade. D'après la convention de signe utilisée (figure 12, la fonction de transfert du correcteur PPF est dans le cas SISO :

$$
H_{l}(s)=-g \frac{1}{1+\frac{2 \xi_{f}}{\omega_{f}} s+\frac{1}{\omega_{f}^{2}} s^{2}}
$$

où $g, \omega_{f}$ et $\xi_{f}$ sont respectivement le gain, la pulsation naturelle et l'amortissement modal choisis pour le correcteur.

Les réglages des trois paramètres $g, \omega_{f}$ et $\xi_{f}$ définissant entièrement le compensateur $H_{l}(s)$ sont réalisés de manière itérative par simulation sur le lieu d'Evans et sur la représentation fréquentielle du transfert de boucle. L'influence de ces paramètres sur l'amortissement sert de base de considérations pour leur réglage :

- d'importantes valeurs du coefficient d'amortissement $\xi_{f}$ conduisent dans la réponse en phase à une région active importante, alors que de faibles valeurs de $\xi_{f}$ améliorent l'amortissement du système précompensé par $H_{l}$. Comme valeur de compromis, il est choisi pour la suite $\xi_{f}=50 \%$;

- après plusieurs essais en simulation, la pulsation naturelle du filtre PPF $\omega_{f}$ est choisie égale à $2258 \mathrm{rad}^{-1}{ }^{-1}$;

- l'influence de la variation de gain sur l'amortissement est reportée sur le lieu des pôles (figure13). Sur les lieux d'Evans reportés ci-dessous, le lieux des pôles du système est étudié en fonction du gain $g$ défini dans l'équation [28] (l'amortissement et la pulsation naturelle ont été fixés par les valeurs déterminées précédemment). Par ailleurs, cette valeur de gain statique, située sur les demi-boucles gauches dans le plan complexe, assure la stabilité du système. Si la structure présente une alternance pôle/zéro (comme optimisée par FlexIn), le lieu d'Evans reste entièrement compris dans le demi-plan complexe gauche, garantissant la stabilité robuste asymptotique (voir le cas du mode 1 de $D(s)$, figure 14 a).

Cependant, les nécessités matérielles de notre chaîne d'instrumentation ne nous permettent plus de conserver cette propriété d'alternance après le deuxième mode vi- 


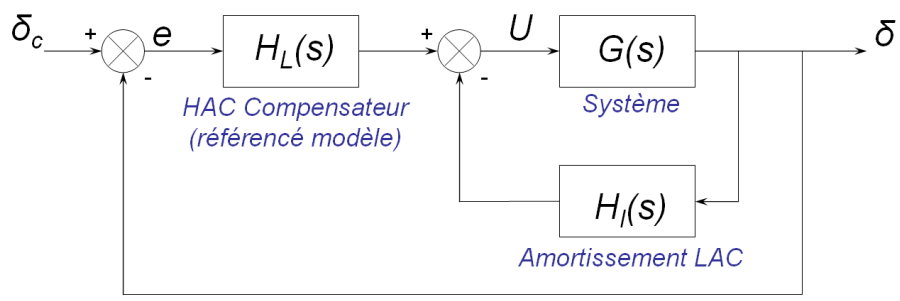

Figure 12. Principe de la commande HAC/LAC

bratoire $\omega_{n 2}$ de $D(s)$, car le filtre antirepliement ajoute un pôle double supplémentaire à $\omega_{F} \approx 6280 \mathrm{rad} / \mathrm{s}$ (figure $14 \mathrm{~b}$ ). Une boucle qui entre dans le domaine d'instabilité se forme alors.

Comme valeur de compromis entre les performances d'amortissement optimal pour le mode 1 (grande valeur de $g$ ) et de stabilité pour le mode 2 (petite valeur de $g$ ), il a été choisi $g=4,7$ (figure 13 ).

De tels paramètres améliorent l'amortissement modal $\xi_{1}$ du système jusqu'à $3,14 \%$ dans la première boucle interne, au lieu de $1,97 \%$ en boucle ouverte. Bien que ces réglages détériorent l'amortissement modal $\xi_{2}\left(\xi_{2}=1,20 \%\right.$ au lieu de $\left.2,12 \%\right)$, la pente de $-40 \mathrm{~dB} /$ decade induite par le correcteur $H_{l}$, puis le second correcteur $H_{L}$ de la boucle externe, diminueront par la suite sensiblement les effets oscillatoires de ce mode (figure15).

\subsection{Synthèse du régulateur HAC}

La synthèse fréquentielle du régulateur $H_{L}$ est réalisée manuellement sur le transfert $G$ pré-compensé par $H_{l}$.

- La valeur du gain en basse fréquence est sensiblement augmentée par l'ajout d'un intégrateur pur dans le correcteur $H_{L}$, permettant ainsi d'éviter les erreurs statiques et de rejeter les perturbations statiques. En hautes-fréquences, cet apport atténue les bruits capteurs et améliore la stabilité.

- L'intégrateur est corrigé par un terme prémultiplicatif pour améliorer la bandepassante $\omega_{B . P .}$ du transfert de boucle $L$, défini comme suit

$$
L(s)=\frac{H_{L}(s) G(s)}{1+H_{l}(s) G(s)},
$$

jusqu'à $\omega_{B . P .} \geq 200 \mathrm{rad} / \mathrm{s}$.

- Un retard de phase de 20 centré sur le deuxième mode résonant complète le régulateur final. Il augmente la marge de gain entre l'amplitude maximale du second pic et la droite à $0 \mathrm{~dB}$, pour empêcher l'apparition d'un régime transitoire qui soit vibratoire, tout en veillant à ne pas détériorer la bande passante. 


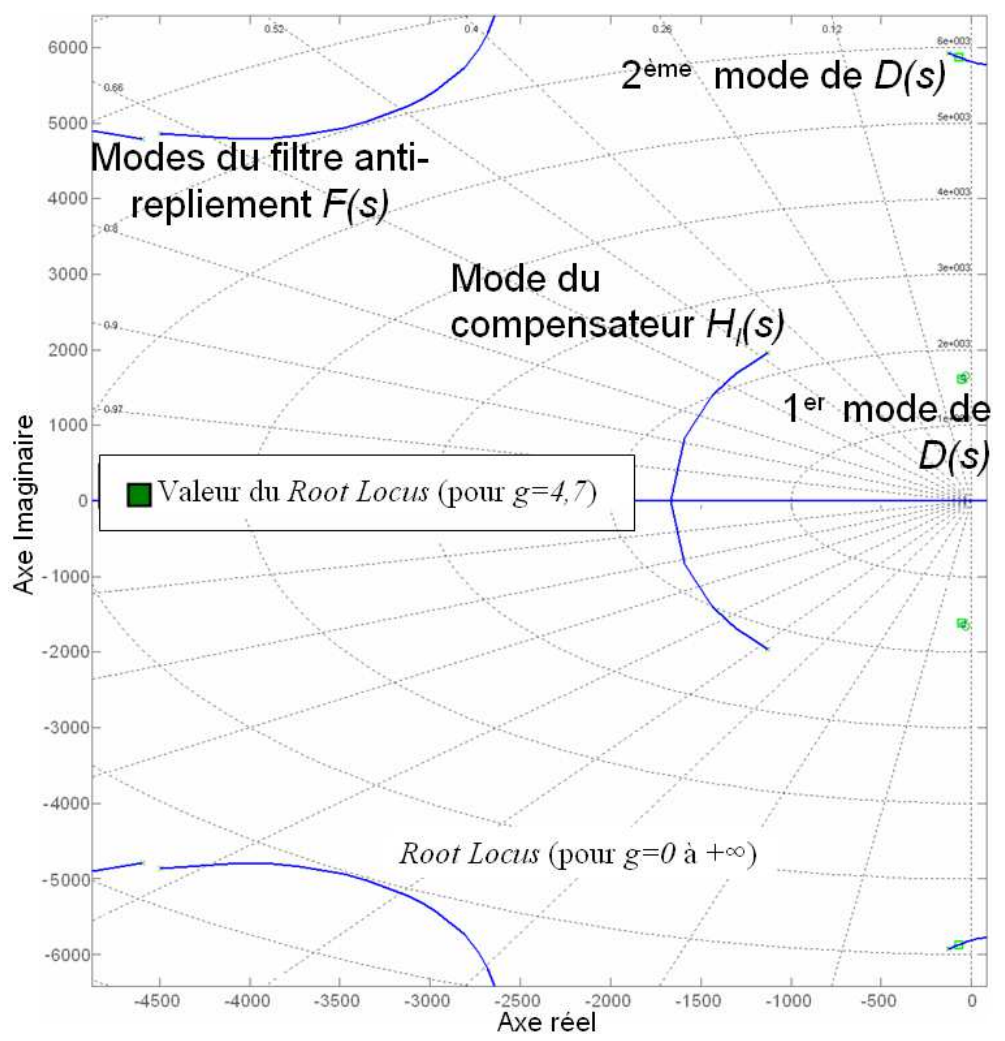

Figure 13. Lieu des pôles en boucle-fermée dans le plan complexe en fonction du gain $g$

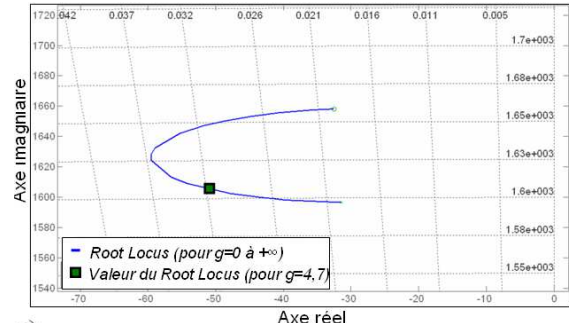

a)

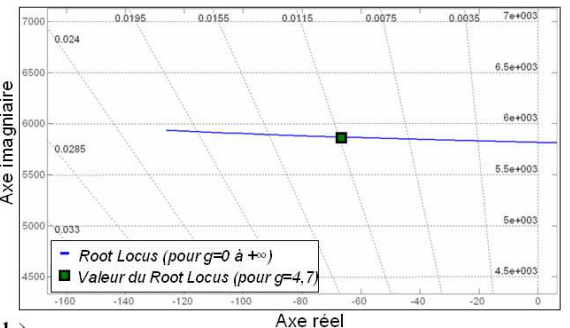

b)

Figure 14. Zooms sur le lieu des pôles de $D(s)$ (a) premier mode, (b) second mode (le graphique est symétrique par rapport à l'axe réel) 


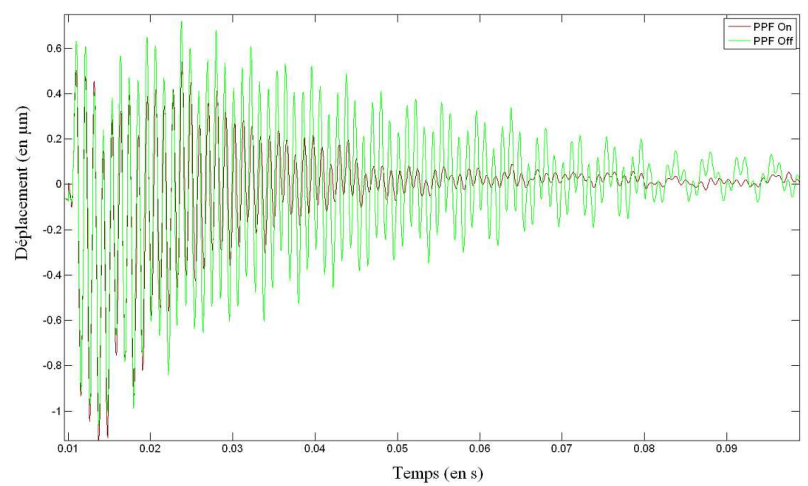

Figure 15. Réponses impulsionnelles expérimentales pour le système libre et compensé par $H_{l}$

Le régulateur HAC ainsi obtenu s'écrit :

$$
H_{L}(s)=2389\left(\frac{1}{s}\right)\left(\frac{1+0.00012 s}{1+0.00024 s}\right)
$$

Il modifie le transfert de boucle $L$ comme indiqué sur la figure 16 garantissant des marges intéressantes : marge de gain $M_{g}=16.60 \mathrm{~dB}$, marges de phase $M_{\varphi}=$ $78.62 \mathrm{deg}$ à $\omega_{P}=217.56 \mathrm{rad} / \mathrm{s}$ et marge de module $M_{m}=16.00 \mathrm{~dB}$ (rayon du cercle centré en $(-1,0)$ et tangent au lieu de Nyquist - robustesse aux variations de la fonction de transfert).

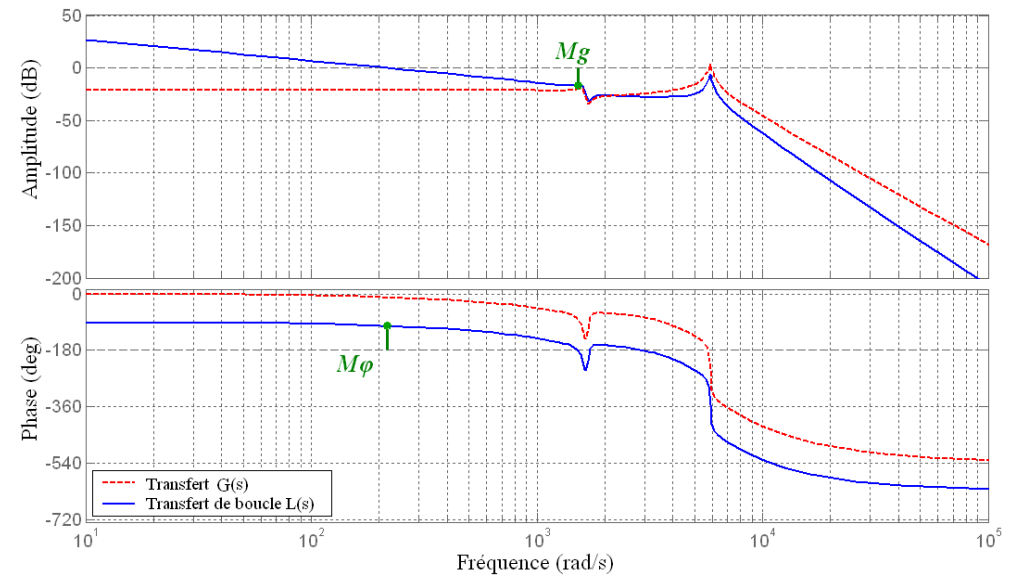

Figure 16. Influence du correcteur HAC/LAC sur le transfert de boucle L et marges de stabilité. 


\subsection{Résultats expérimentaux}

Ce régulateur a été implémenté pour commander le micro-actionneur. La figure 17 présente sa réponse expérimentale à une consigne en échelon. Les performances en asservissement ne présentent pas de dépassement, et le temps de réponse est estimé à $t 5 \%=9,9 \mathrm{~ms}$, conformément au résultat de simulation. À titre de comparaison, le temps de réponse en boucle ouverte du système est estimé à $t_{5 \%}=33,5 \mathrm{~ms}$.

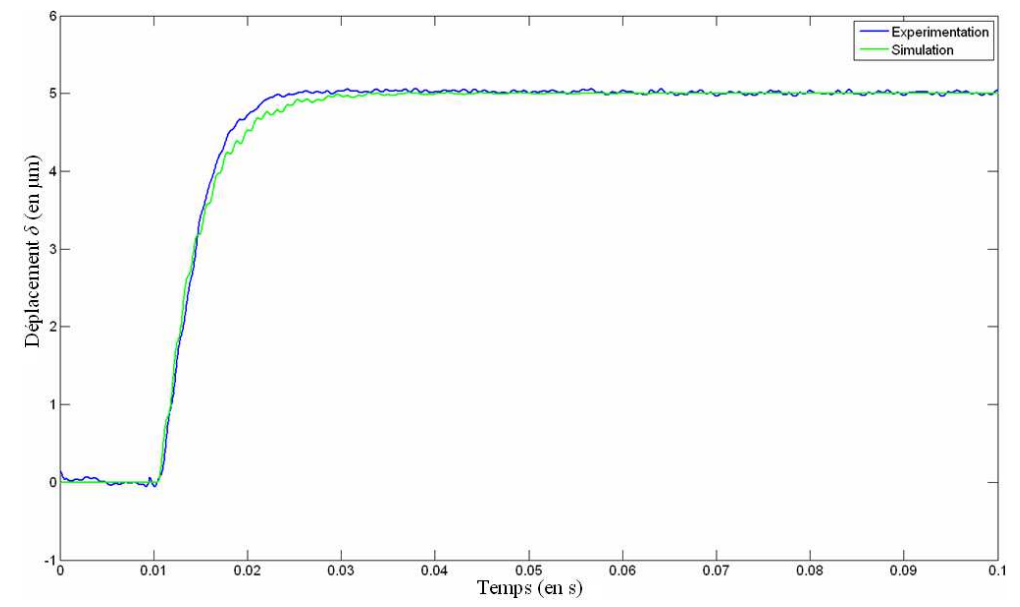

Figure 17. Réponses indicielles expérimentale et simulée en asservissement pour le micro-actionneur commandé par le régulateur HAC/LAC (échelon consigne d'amplitude $5 \mu \mathrm{m})$

\section{Conclusions}

Une nouvelle méthode d'aide à la conception optimale de systèmes microrobotiques à déformations réparties et à actionnement piézoélectrique intégré a été présentée. Elle est basée sur l'agencement optimal, grâce à un algorithme génétique multicritère, de blocs flexibles élémentaires, de types treillis de poutres, décrits par une méthode aux éléments finis dans un domaine de conception fixé. Ces blocs élémentaires peuvent être passifs ou rendus actifs par effet piézoélectrique inverse. La fonction d'actionnement est donc totalement intégrée dans la structure même du mécanisme, permettant de se rapprocher du concept de système robotique monolithique.

En outre, une nouvelle représentation dynamique (réponse fréquentielle en boucle ouverte) du comportement entrée(s)-sortie(s) des mécanismes flexibles a été introduite dans la méthode de conception optimale. Elle permet la prise en compte, dès la phase amont de conception, de nouvelles spécifications du cahier des charges, directement liées aux performances de commande ultérieure des systèmes synthétisés. Ainsi, deux 
nouveaux critères d'optimisation ont été implémentés dans le logiciel FlexIn. Lorsqu'ils sont considérés dans le problème de synthèse, ces critères visent à faciliter à terme l'identification et la commande ultérieures des systèmes générés. Ces critères ont été mis à profit pour la conception d'une nouvelle micropince piézoélectrique monolithique, capable de réaliser à terme des tâches de micromanipulation.

La micropince pseudo-optimale synthétisée a été prototypée et testée. Tirant profit des propriétés optimales de la réponse fréquentielle en boucle ouverte du système, la synthèse d'un correcteur basé sur des techniques d'amortissement actif (type PPF), corrigé par une action proportionnelle-intégrale, donne des performances satisfaisantes et des marges de robustesse très intéressantes. Cette approche est d'autant plus attrayante que la synthèse d'un tel correcteur par le lieu d'Evans reste relativement simple, et peut garantir une stabilité asymptotique sous réserve de certaines propriétés structurelles sur la réponse fréquentielle du système (alternance pôle/zéro).

Un autre intérêt majeur de cette approche HAC/LAC est l'ordre réduit du correcteur synthétisé (ordre 4), comparativement aux approches classiques de commande robuste (synthèse $H_{\infty}$, placement de pôles robustes RST par exemple). La méthodologie employée ici (à partir du lieu d'Evans pour le régulateur LAC et du transfert de boucle pour le régulateur HAC) est plus aisée à mettre en œuvre si le concepteur de la loi de commande n'est pas familier avec ces outils de robustesse. Les régulateurs obtenus avec de telles méthodes amènent, à moins d'une étape de réduction de modèles a posteriori, à des correcteurs d'ordre élevés. Par ailleurs, nous avons montré dans (Grossard, 2008) que les performances en précision et stabilité atteintes par placement de pôles robuste (RST) et commande $H_{\infty}$ sont similaires à celles atteintes par le régulateur $\mathrm{HAC} / \mathrm{LAC}$ proposé.

Dans la perspective de conception de systèmes adaptroniques, et à terme d'intégration du correcteur dans des systems-on-chip, l'approche HAC-LAC justifie alors pleinement son intérêt.

\section{Bibliographie}

Agnus J., Nectoux P., Chaillet N., « Overview of Microgrippers and Design of a Micromanipulation Station Based on a MMOC Microgripper $»$, Proceedings of the IEEE International Symposium on Computational Intelligence in Robotics and Automation (CIRA), Espoo, Finland, p. 117-123, June 27-30, 2005.

Allaire G., Jouve F., Toader A.-M., « Structural optimization using sensitivity analysis and a level set method », J. Comp. Phys., vol. , p. 363-393, 2004.

Aphale S. S., Fleming A. J., Moheimani S. O. R., « Integral resonant control of collocated smart structures », Smart Materials and Structures, vol. 16, p. 439-446, 2007.

Aubrun J. N., Lyons M. G., Ratner M. J., « Structural control for a circular plate », J. Guid. Contr. Dynam., vol. 7, p. 535-545, October, 1984.

Bendsœ M. P., Sigmund O., Topology optimization : theory, methods and applications, Springer Verlag, 2003. 
Bernardoni P., « Outils et méthodes de conception de structures mécaniques à déformations réparties et actionnement discret - applications en microrobotique », Thèse de l'Université de Paris VI, 2004.

Canfield S., Frecker M., « Topology Optimization of Compliant Mechanical Amplifiers for Piezoelectric Actuators », Structural and Multidisciplinary Optimization, vol. 20, p. 269278, 2000.

Croft D., Devasia S., « Hysteresis and vibrations compensation for piezoactuator », AIAA Journal, vol. 21, p. 710-717, 1998.

Dimmler M., Holmberg U., Longchamp R. L., « Hysteresis compensation of piezoactuator », proc. 5th European Control Conf., 1999.

Gawronski W., « Discrete-time norms of flexible structure », Int. Journal of Sound and Vibration, vol. 264, p. 983-1004, 2003.

Girard A., « Dynamique des structures - Techniques d'analyse et d'essai », Dossier Techniques de l'Ingénieur, 1997.

Grossard M., « Contribution à la conception optimale et la commande de systèmes mécatroniques flexibles à actionnement piézoélectrique intégré - Application en microrobotique », Thèse de l'Université de Franche-Comté, 2008.

Grossard M., Rotinat-Libersa C., Chaillet N., Boukallel M., « Mechanical and control-oriented design of a monolithic piezoelectric microgripper using a new topological optimisation method », IEEE/ASME Transactions on Mechatronicsp. 32-45, 2008.

Grossard M., Rotinat-Libersa C., Chaillet N., Perrot Y., « Flexible building blocks method for the optimal design of compliant mechanisms using piezoelectric material », 12th IFToMM World Congress in Mechanism and Machine Science, Besançon, France, June 18-21, 2007.

Hac A., Liu L., « Sensor and actuator location in motion control of flexible structures », Journal of Sound and Vibrations, vol. 167, p. 239-261, 1993.

Hurlebausa S., Gaul L., « Smart structure dynamics », Mechanical Systems and Signal Processing, vol. 20, p. 255-281, 2006.

Janocha H., Adaptronics and Smart Structures - Basics, Materials, Design and Applications, Second Edition Springer Berlin Heildelberg New-York, 2007.

Lim K. B., Gawronski W., Actuators and sensor placement for control of flexible structures in Control and Dynamics Systems : Advances in Theory and Applications, ed. London, Academic Press, 1993.

Martin G. D., « On the control of flexible mechanical systems », PhD Dissertation, Stanford University, USA, 1978.

Missiles L., Inc. S. C., «Vibration control of space structures : a high and low autorithy hardware implementation », AFWAL-TR-83-3074, July, 1983.

Moore B. C., « Principal component analysis in linear systems : controllability, observability, and model reduction », IEEE Trans. on Automatic Control, vol. 26, p. 17-32, 1981.

Pons J. L., Emerging actuator technologies : a micromechatronic approach, Wiley, 2005.

Preumont A., Vibration control of active structures : an introduction, 2nd edition, Kluwer academic publishers, 2002.

Rakotondrabe M., « Développement et commande modulaire d'une station de micromanipulation », Thèse de doctorat, LAB - CNRS Université de Franche-Comté, 2006. 
Vincent S., « Etude de la complémentarité d'actionneurs pour la commande active des structures flexibles », Thèse de doctorat de l'Ecole Nationale Supérieure de l'Aéronautique et de l'Espace, 1996. 


\section{ANNEXE POUR LE SERVICE FABRICATION A FOURNIR PAR LES AUTEURS AVEC UN EXEMPLAIRE PAPIER DE LEUR ARTICLE ET LE COPYRIGHT SIGNE PAR COURRIER LE FICHIER PDF CORRESPONDANT SERA ENVOYE PAR E-MAIL}

1. ARTICLE POUR LA REVUE :

662 JESA - 44/2010. Commande dans le micromonde

2. Auteurs :

Mathieu Grossard ${ }^{*}$ - Nicolas Chaillet ${ }^{* *}$ - Mehdi Boukallel ${ }^{*}$ Christine Rotinat-Libersa* — Arnaud Hubert ${ }^{* *}$

3. TITRE DE L'ARTICLE :

Synthèse et commande robuste d'une micropince piézoélectrique intégrée

4. TITRE ABRÉGÉ POUR LE HAUT DE PAGE MOINS DE 40 SIGNES :

Synthèse et commande d'une micropince

5. DATE DE CETTE VERSION :

23 juin 2010

6. CoOrdonnéEs des Auteurs :

- adresse postale :

* CEA, LIST, Interactive Robotics Laboratory, Fontenay aux Roses, F92265, France

mathieu.grossard@cea.fr

** Institut Femto-ST, UMR CNRS 6174 -UFC/ENSMM/UTBM, Département Automatique et Systèmes Micro-Mécatroniques, 24 rue Alain Savary, F-25000 Besançon

- téléphone : 0146549188

- télécopie : 0146548980

- e-mail : mathieu.grossard@cea.fr

7. LOGICIEL UTILISÉ POUR LA PRÉPARATION DE CET ARTICLE :

${ }_{\mathrm{AT}} \mathrm{T} X$, avec le fichier de style article-hermes.cls, version $1.27 \mathrm{du}$ 07/09/2006.

8. FORMULAIRE DE COPYRIGHT :

Retourner le formulaire de copyright signé par les auteurs, téléchargé sur : http://www.revuesonline.com 